\title{
Cognitive Remediation in
} Middle-Aged or Older Inpatients with Chronic Schizophrenia: A Randomized Controlled Trial in Korea

\author{
Kee-Hong Choi ${ }^{1}$, Jinsook Kang ${ }^{1}$, Sun-Min Kim ${ }^{1}$, Seung-Hwan Lee ${ }^{2}$, Seon-Cheol Park ${ }^{3}$, \\ Won-Hye Lee ${ }^{4}$, Sun $\mathrm{Choi}^{5}$, Kiho Park ${ }^{1}$ and Tae-Yeon Hwang ${ }^{6 *}$ \\ ${ }^{1}$ Department of Psychology, Korea University, Seoul, South Korea, ${ }^{2}$ Department of Psychiatry, Inje University College of \\ Medicine and Ilsan Paik Hospital, Goyang, South Korea, ${ }^{3}$ Department of Psychiatry, Inje University College of Medicine and \\ Haeundae Paik Hospital, Busan, South Korea, ${ }^{4}$ Department of Clinical Psychology, National Center for Mental Health, Seoul, \\ South Korea, ${ }^{5}$ Department of Clinical Psychology, Yongin Mental Hospital, Yongin, South Korea, ${ }^{6}$ Division of Mental Health \\ Service and Planning, National Center for Mental Health and Yongin WHO Collaborating Center for Psychosocial \\ Rehabilitation and Community Mental Health, Seoul, South Korea
}

OPEN ACCESS

Edited by:

Tomiki Sumiyoshi,

National Center of Neurology and

Psychiatry (Japan), Japan

Reviewed by:

Satoru Ikezawa,

Japan Agency for Medical Research and Development, Japan Mahesh Menon, University of British Columbia, Canada

*Correspondence: Tae-Yeon Hwang lilymh@gmail.com

Specialty section: This article was submitted to Psychopathology, a section of the journal

Frontiers in Psychology

Received: 20 October 2017 Accepted: 27 December 2017 Published: 06 February 2018

Citation:

Choi K-H, Kang J, Kim S-M, Lee S-H,

Park S-C, Lee W-H, Choi S, Park K and Hwang T-Y (2018) Cognitive Remediation in Middle-Aged or Older Inpatients with Chronic Schizophrenia: A Randomized Controlled Trial in Korea. Front. Psychol. 8:2364. doi: 10.3389/fpsyg.2017.02364
Background: Accumulating evidence indicates that cognitive remediation (CR) is effective for improving various cognitive deficits in adult patients with schizophrenia. Although reports of brain plasticity in older adults and the service needs for chronic patients with schizophrenia are increasing, very few randomized controlled trials of CR have been conducted in middle-aged or older inpatients with chronic schizophrenia. We investigated the efficacy of individualized CR on the cognitive impairments of middle-aged or older inpatients with chronic schizophrenia within the context of comprehensive psychiatric rehabilitation (PR) by comparing the results obtained with PR only and treatment as usual (TAU).

Method: Fifty-seven middle-aged and older individuals with chronic schizophrenia and mild to moderate cognitive deficits were enrolled. Thirty-eight who were undergoing PR were randomly assigned to $\mathrm{CR}+\mathrm{PR}(N=19)$ or PR-only $(N=19)$ groups. Nineteen participants who were undergoing TAU without CR or PR were evaluated pre- and post-treatment.

Results: CR was easily provided and well received (drop-out rates $=5.3 \%$ ) by middle-aged or older psychiatric inpatients. Compared to the PR-Only or TAU patients, patients in the CR + PR group showed greater improvement in executive functioning. Compared to TAU patients, CR + PR and PR-only patients showed greater improvement in logical memory. More patients in the $\mathrm{CR}+\mathrm{PR}$ group improved clinically significantly in executive functioning and logical memory, compared with the PR-only and TAU patients.

Conclusions: These results suggested that $\mathrm{CR}$ improved some cognitive deficits in middle-aged or older inpatients with chronic schizophrenia and that it was effective as an adjunctive treatment to the usual PR services provided in inpatient settings.

\section{Clinical Registration: KCT0002609}

Keywords: cognitive remediation, older patients, brain plasticity, schizophrenia, inpatient psychiatric rehabilitation 


\section{INTRODUCTION}

Cognitive deficits have been identified as a key predictor of the functioning of patients with schizophrenia across various phases of the illness from the first episode to chronic illness, even after considering the patients' psychiatric symptoms (Green, 1996; Green and Nuechterlein, 1999; Green et al., 2004; Kurtz et al., 2005; Keefe et al., 2006; Rund et al., 2007). Cognitive functioning has critical therapeutic implications in diverse contexts, including treatment (Smith et al., 1999; Lysaker and Buck, 2007), and vocational rehabilitation (Bell et al., 2007, 2014; McGurk et al., 2015).

Given the therapeutic and functional importance of cognitive deficits in this population (Keefe and Harvey, 2012), cognitive remediation (CR) has received substantial attention as an adjunct treatment option for psychiatric rehabilitation (PR) (Wykes and Spaulding, 2011). In recent decades, randomized controlled trials (RCTs) have demonstrated the efficacy of CR on psychiatric symptoms, cognition, and functioning (Krabbendam and Aleman, 2003; McGurk et al., 2007a; Grynszpan et al., 2011; Wykes et al., 2011).

With the recent increases in life expectancy, the service needs for chronic mid-aged or older adults with chronic schizophrenia are growing (Granholm et al., 2005; Bartels et al., 2014; Schoepf et al., 2014). Cognitive function is responsible for functional competence even after considering key demographic information, such as age and education, and the anticholinergic burden of medications, in older adults with schizophrenia (Tsoutsoulas et al., 2016).

Even though older adults have brain plasticity (Willis et al., 2006; Boron et al., 2007), it is unclear whether CR is effective in middle-aged or older adults with or without schizophrenia (Kontis et al., 2013) or the benefits of CR are also produced by untrained cognitive tasks (Owen et al., 2010).

Even though CR has been reported as more beneficial to younger patients with schizophrenia compared to mid-aged or older patients with schizophrenia (McGurk and Mueser, 2008; Kontis et al., 2013), several studies have indicated that CR still has benefits for in mid-aged or older patients. Wykes and colleagues (Wykes et al., 2007) have reported that, after approximately $30 \mathrm{~h}$ of cognitive training, patients who were 40 or older had similar improvements in their memory as those shown by patients younger than 40. Bowie et al. (2014) have reported that older patients (mean age, 45.4) showed improvements in verbal memory and verbal fluency as younger patients (mean age, 28.1) did, while only the younger patients had improvements in processing speed and executive functioning. Most recently, Corbera et al. (2017) conducted a secondary analysis of the results of three different RCTs to investigate the responses of patients with three different age ranges (i.e., $<26$, between 26 and 39, and over 40) to CR treatment, and they reported that the younger

Abbreviations: CL, Conceptual level; CR, Cognitive remediation; LM, Logical memory; PANSS, Positive and negative syndrome scale; PR, Psychiatric rehabilitation; RCI, Reliable change index; RCTs, Randomized controlled trials; TAU, Treatment as usual; WAIS-IV, Wechsler Adult Intelligence Scale-Fourth edition; WCST, Wisconsin card sorting test; WMS-IV, Wechsler Memory ScaleFourth Edition. patients benefited more (trend level of significance) compared with the older patient group.

Because promising findings have been reported in non-RCTs, a RCT of the role of CR in the treatment of the cognitive functioning of middle-aged or older patients with schizophrenia is still needed. Sharma et al. (2016) highlighted in their review that controlled studies are needed to better understand whether CR benefits older adults with severe mental illness within the context of broad PR treatments, even though CR appears to have therapeutic benefits for older adults with various health conditions, such as mild cognitive impairment, brain injury, and severe mental illness (Bartels and Pratt, 2009).

To the best of our knowledge, only one RCT study has been conducted to date in middle-aged or older out-patients with schizophrenia (mean age, 46.9 and 48.5 for CR and Control, respectively), and it reported null effects in the neuropsychological assessments of the outpatients compared to active control groups (Dickinson et al., 2010). Since Spaulding et al. (1999a)'s seminal work on CR for inpatients with schizophrenia during intensive PR, numerous RCT trials have demonstrated its clinical effectiveness and functional importance for recovery of relatively younger inpatients with schizophrenia (Medalia et al., 1998, 2000, 2001; Sartory et al., 2005; Silverstein et al., 2005, 2009; Ueland and Rund, 2005; Vauth et al., 2005; Wykes et al., 2007). Despite the growing number of chronic and older inpatients with schizophrenia, only one RCT has been conducted on CR in relatively older in-patients (mean age, 43.46), and its findings suggested that CR results in additional benefits within the context of vocational rehabilitation (Lindenmayer et al., 2008). Previous meta-analyses (McGurk et al., 2007b; Wykes et al., 2011) have emphasized that investigations of whether CR has additive benefits in older patients with schizophrenia within the context of PR are of great interest (Bartels and Pratt, 2009; Sharma et al., 2016).

To examine the effects of $\mathrm{CR}$ and PR together and PR only on neurocognitive functioning and psychiatric symptoms in this study, we included a treatment-as-usual (TAU) group (without PR) as another control group. Importantly, to the best of our knowledge, no RCTs have been conducted on non-western inpatients within the context of comprehensive PR, even though inpatient clinics, in conjunction with community-based PR, are important settings for PR.

Thus, in the current study, we employed a RCT design (CR + PR vs. PR only) to evaluate the efficacy of a CR program for middle-aged or older inpatients with chronic schizophrenia within a PR context compared to TAU. We hypothesized that $\mathrm{CR}+\mathrm{PR}$ treatment would result in the greatest improvements, followed by PR only and TAU, in untrained neurocognitive domains and psychiatric symptoms in middle-aged or older inpatients with chronic schizophrenia.

\section{METHODS}

\section{Clinical Trial Design}

Of the 79 inpatients with schizophrenia who were referred to this clinical trial $(53$ from the PR unit and 26 from the TAU unit), eight did not meet the inclusion criteria and 14 declined 
to participate. A final total of 38 inpatients with schizophrenia from the PR unit who met the inclusion criteria of the study and who gave consent were randomly assigned to the CR + PR or PRonly groups (Figure 1). Nineteen inpatients with schizophrenia from the TAU unit were allocated to the TAU group (Figure 1). The participants from the PR unit who met the study criteria and consented to the current trial were informed that they were assigned to $\mathrm{CR}$ in addition to the usual PR programs or the usual PR program only. The TAU group was informed that they would be assessed two different times. All participants were interviewed and assessed before starting the trial and immediately after the trial. Positive and Negative Syndrome Scale (PANSS) and neurocognitive assessments were conducted by master-level pretrained research assistants. The administration and scoring procedures were supervised by licensed clinical psychologists (K. H. Choi, W. H. Lee, and S. Choi). The current study has been registered with Clinical Research Information Service (CRIS) registry, number KCT0002609.

\section{Participants}

The diagnoses of the 57 inpatients with schizophrenia (32 males and 25 females) were confirmed by the Structured Clinical Interview for DSM-IV Axis I disorders (American Psychiatric Association, 1994). Prior to the very recent reform in "Acts on the Improvement of Mental Health and the Support for Welfare Services for Mental Patients" in Korea, the average percentage of involuntary hospitalization to mental health units in Korea has been very high from 67.9 to 93.3\% (Ministry of Health Welfare, 2016). All participants in this study were involuntarily admitted to the long stay close unit of the hospital. The mean age of the patients was 50.07 , with a range of 36-59, and their number of years of education was 10.93. All patients had been on stable medication regimens for the previous 30 days, with no groups differed for psychotropic medication dose equivalents (Inada and Inagaki, 2015) and maintained their medication dosages during the current 3-month long outcome trial, except for PRN medications. Participants were excluded if they had any of the following: substance use, serious traumatic brain injury or other neurological disorder, or acute psychiatric symptoms. Four patients were dropped due to discharge. All participants in this research voluntarily participated and provided written informed consent.

\section{Measures}

\section{Psychiatric Symptoms}

Psychiatric symptoms were measured by using the PANSS (Kay et al., 1987), which includes five subscales: negative, excitement, cognitive, positive, and depressive (Bell et al., 1994). The internal consistency coefficients of the original version of each subscale were the following: negative ( $\alpha=0.86)$, excitement $(\alpha=0.76)$, cognitive $(\alpha=0.79)$, positive $(\alpha=0.80)$, and depressive $(\alpha=$ 0.69 ). In the current study, the internal consistency coefficients were of each subscale were the following: negative $(\alpha=0.92)$, excitement ( $\alpha=0.45)$, cognitive $(\alpha=0.43)$, positive $(\alpha=0.74)$, and depressive $(\alpha=0.67)$. In the current study, the inter-rater reliability coefficient was 0.96 .

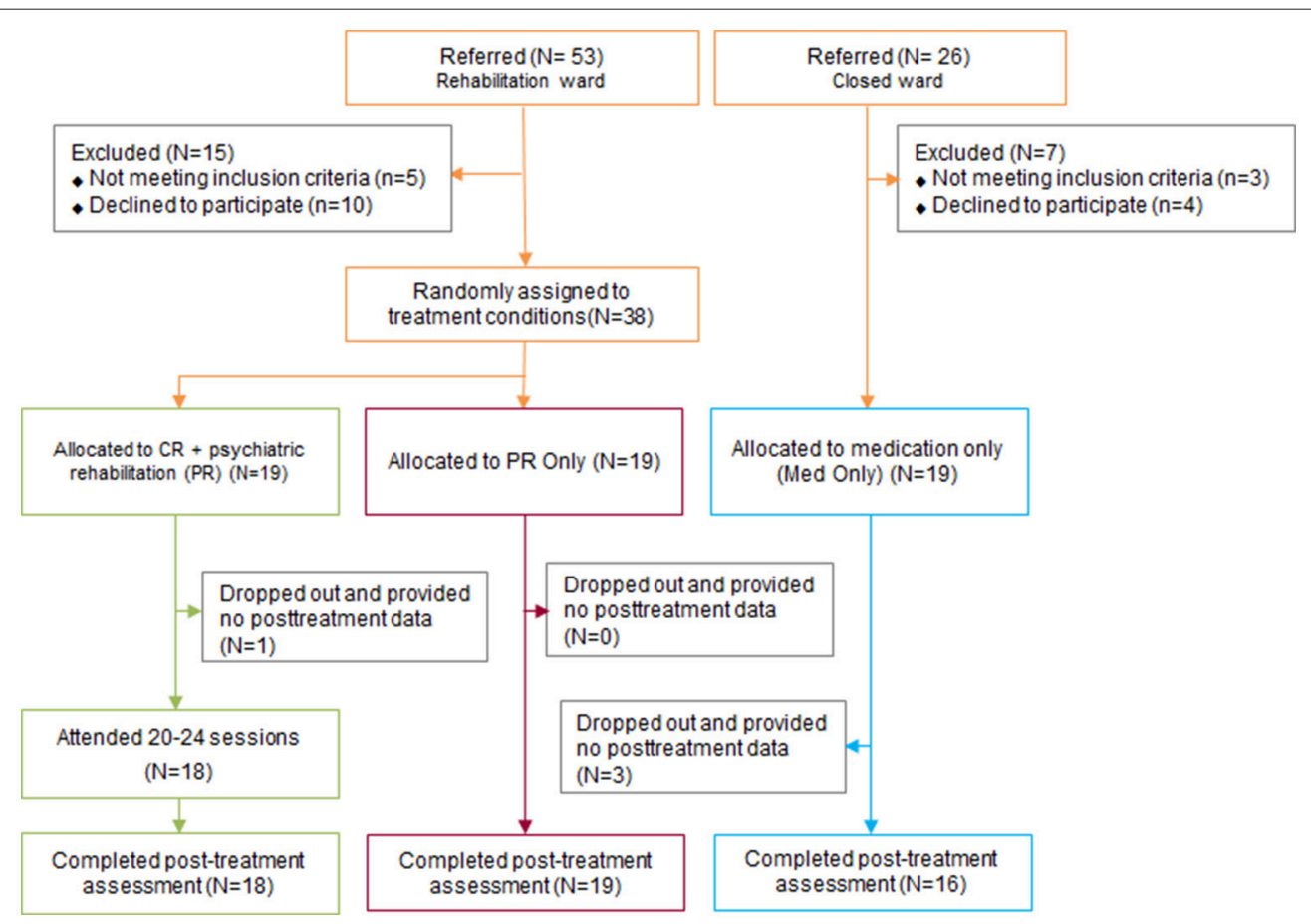

FIGURE 1 | Consolidated standards of reporting trials diagram of the selection of the participants in the study. CR, Cognitive remediation; PR, Psychiatric rehabilitation; TAU, Treatment As Usual. 


\section{Motivation}

Participants' overall motivation was measured by the BIS/BAS scale (Carver and White, 1994; Kim and Kim, 2001).

\section{Premorbid IQ}

Premorbid IQ was estimated by using the Information subtest score from the Wechsler Adult Intelligence Scale-Fourth edition (WAIS-IV) (Wechsler, 2008) and demographic variables, including years of education, age, gender, and ethnicity (Kim et al., 2015).

\section{Neurocognition}

Attention/processing speed was assessed by using the Trail Making Test A, which requires the examinee to draw a line between numbers in order within $360 \mathrm{~s}$ (Arbuthnott and Frank, 2000). Processing speed was also assessed by using the symbolcoding test, which is a subtest of the WAIS-IV (Wechsler, 2008) and which requires the conversion of numbers to matched symbols in a limited amount of time. Patients with schizophrenia exhibit decreased processing speed and working memory (Kreiner and Ryan, 2001).

Working memory was assessed by using the letter-number sequencing test, which is a subtest of the WAIS-IV (Wechsler, 2008) and which requires the manipulation or visualization of orally presented words and numbers and the recitation of the numbers first in numerical order and then letters in alphabetical order. Verbal logical memory (LM) was assessed by using the LM (I and II) tests, which are subtests of the Wechsler Memory Scale-Fourth Edition (WMS-IV) (Wechsler, 2009). The test is comprised of immediate recall, delayed recall, and recognition tasks for two stories. The stories are read with clear pronunciation by the examiner. The LM tests measure the examinees' verbal memory capacity, which is the ability to recall organized and meaningful linguistic information components.

Executive functioning was assessed by using the Trail Making Test B (Trails B), Wisconsin Card Sorting Test (WCST) 64, and Verbal Fluency Test. The Trails B requires the examinee to draw a line between numbers and letters that are scattered on paper following the given rules within $360 \mathrm{~s}$ (Arbuthnott and Frank, 2000). The WCST evaluates executive function, which is the essential cognitive function that enables goal setting, planning, and goal-directed behaviors. The WCST 64 consists of four stimulus cards and 64 response cards that have three different dimensions (i.e., form, color, and number), with each having four components. The examinee is required to place each response card under a stimulus card that they consider a match. After matching the cards, (s)he gets feedback on whether they were right or wrong. Because they are not given any instructions on the pairing rules, the examinees have to determine the rules from the feedback. The verbal fluency test requires the examinees to find as many words as possible that begin with a given consonant in a limited amount of time (60 s) (Lee et al., 2000).

\section{CR}

The CR treatment consisted of 24 sessions that occurred twice a week for $1 \mathrm{~h} /$ session for over 3 months. The PSSCogRehab software program (version 12.0; Psychological Software Services,
Inc., Indianapolis, IN, USA) was translated in Korean by K. H. Choi's research group and then used for CR training (Bracy, 2012). The CR training was formulated to include practicing attention, memory, and executive functioning (Figure 2). The starting level and initial training schedules were determined for each individual by a therapist according to their pretreatment assessment. The training schedules (e.g., targeted neurocognitive domains, difficulty levels, etc.) were individualized and updated based on the participants' preferences and levels of performance. CR games from Lumosity.com were also employed to supplement the CR training for spatial memory (e.g., tile matrix and treasures on the beach) and executive functioning (e.g., color match and world of illusion). Because most participants in the current study were not familiar with using computers, the therapists introduced the basic skills (e.g., using a mouse) of computer usage at the initial session and then gradually moved to the $\mathrm{CR}$ tasks. The CR sessions consisted of computer-based CR training (50 $\mathrm{min})$ and a bridging group session (10 $\mathrm{min}$ ) (Medalia et al., 2009). To increase motivation, the participants were reminded at the beginning of each session of their goals for joining the CR program, and they were given the opportunity to link their personal goals with the CR training. In addition, the participants were able to choose tasks that they wanted to practice in each session with the clinician's assistance and monitor their progress. After about $50 \mathrm{~min}$ of computer-based CR training, the group members gathered for a bridging group (Medalia et al., 2009), in which they discussed the connections between their goals and the cognitive training and exchanged strategies. The therapists used motivational interviewing techniques to enhance the participants' intrinsic motivation toward the CR (Fiszdon et al., 2016; Lee et al., 2017). All CR sessions were reviewed by K. H. Choi in a weekly supervision meeting or in vivo training sessions to confirm the consistency of the CR protocol.

\section{PR}

The participants who were randomized to the $\mathrm{CR}+\mathrm{PR}$ and PR-only groups received comprehensive inpatient PR, including optimal pharmacotherapy, vocational rehabilitation, social skills training, daily living skills training, illness management, independent living skills training, and patient empowerment program. The PR did not include specific training on neurocognitive functioning.

\section{TAU}

The participants who were recruited from the TAU unit received optimal pharmacotherapy, psychoeducation, socialization and recreational programs. The TAU group did not receive specific training on neurocognitive functioning or PRspecific components, such as vocational rehabilitation or skills training.

\section{Data Analysis}

A series of repeated-measures analyses of variance [withinsubjects factor, time; between-subjects factor, group $(\mathrm{CR}+\mathrm{PR}$, PR only, or TAU)] were conducted to examine the effects of CR on the neurocognitive measures, psychiatric symptoms, and clinic unit behaviors. The effect sizes were calculated with the 


\begin{tabular}{|c|c|c|}
\hline & Training Emphases & Examples of Specific Training Exercises \\
\hline 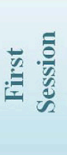 & $\begin{array}{l}\text { - Engagement and rapport } \\
\text { - Program and computer orientation } \\
\text { - Motivation enhancement } \\
\text { - Mouse skills training }\end{array}$ & \\
\hline 最曷 & $\begin{array}{l}\text { - Engagement and rapport (cont.) } \\
\text { - Motivation enhancement (cont.) } \\
\text { - Focused attention } \\
\text { - Divided attention }\end{array}$ & $\begin{array}{l}\text { - Simple Visual/Auditory Reaction: Click the screen when the } \\
\text { visual/auditory stimulus is presented } \\
\text { - Simple Choice Visual Reaction: Click the screen only when } \\
\text { the target visual/auditory stimulus is presented, with random } \\
\text { distracters presented }\end{array}$ \\
\hline 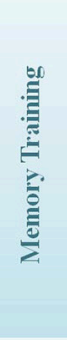 & $\begin{array}{l}\text { - Spatial memory } \\
\text { - Working memory } \\
\text { - Concentration }\end{array}$ & $\begin{array}{l}\text { Digit \& Graphic: Memorize the presented sequential stimuli } \\
\text { (digits or graphics) and recall them in order after pause. The } \\
\text { order of recall can be forward or backward. } \\
\text { - Tile matrix }{ }^{*} \text { : Remember the colorized cells on } 4 \times 4 \text { to } 16 \times 16 \\
\text { matrix. The number of cell on matrix increases when the } \\
\text { colorized cells were identified correctly. } \\
\text { - Treasures on the beach*: Watch and memorize the graphics } \\
\text { (treasures) on screen, and after the graphics are gone, } \\
\text { distinguish items whether they were presented or not. }\end{array}$ \\
\hline 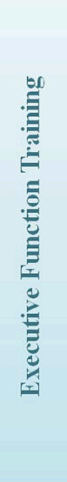 & $\begin{array}{l}\text { - Planning } \\
\text { - Principle learning \& applying } \\
\text { - Effective performance } \\
\text { - Inhibit responses }\end{array}$ & $\begin{array}{l}\text { - Color match }{ }^{*} \text { Two colored words are presented on the } \\
\text { screen and the participant have to respond whether the meani } \\
\mathrm{ng} \text { of the word on left side refers to the color of the word on } \\
\text { right side or not. } \\
\text { - Pyramid: There are three rods and a number of disks of } \\
\text { different sizes. The participant have to move the disks from a } \\
\text { rod to another with minimum movement. Only one disk can } \\
\text { be moved at a time, and no disk can be placed over a smaller } \\
\text { disk. } \\
\text { - World of Illusion*: Puzzle game that each piece has a } \\
\text { colored symbol on it. The horizontal pieces have to be put by } \\
\text { the piece with same figured symbol, the vertical pieces have } \\
\text { to be put by the pieces with the same colored symbol. }\end{array}$ \\
\hline
\end{tabular}

- Participants were able to proceed to next task when they met pass-fail criteria for each task.

- Tasks indicated by star $\left.{ }^{*}{ }^{*}\right)$ refers to the exercises in Lumosity.

FIGURE 2 | Targeted domains and detailed training tasks in CR.

Time-by-Group interaction. Post-hoc analyses were conducted to compare the group means at each time point. To estimate the clinical significance, the reliable change index (RCI) was calculated and compared among the groups (Wise, 2004). The RCI was calculated as $\frac{X_{1}-X_{2}}{S E}\left(\mathrm{X}_{1}=\right.$ pretest score; $\mathrm{X}_{2}=$ post-test score; $\mathrm{SE}=s_{1} \sqrt{1-r_{\mathrm{xx}}} ; \mathrm{s}_{1}=$ the standard deviation of the control group, normal population, or pretreatment group; and $\mathrm{r}_{\mathrm{xx}}=$ the test-retest reliability). RCI scores that were equal or $>1.96$ are considered reliable changes (Wise, 2004).

\section{Power Analysis}

Using $G^{*}$ Power (version 3.1.9.2, Heinrich Heine University, Düsseldorf, Germany) (i.e., repeated measures analyses of variance, measures at two-time points for three groups, $p<$ 0.05 , correlations $=0.5-0.8$, and Cohen's $d=0.58$ in the context of PR) (Faul et al., 2009), moderate effects were found in 45 participants. Considering the potential for missingness, we assigned 19 participants to each group.

\section{Ethical Standards}

The study was approved by both Yongin Mental Hospital Institutional Review Board and Korea University Institutional Review Board. The authors assert that all procedures contributing to this work comply with the ethical standards of the relevant national and institutional committees on human experimentation and with the Helsinki Declaration of 1975, as revised in 2008.

\section{RESULTS}

As shown in Table 1, 38 participants were randomized to either the CR + PR or PR-only groups, and an additional 19 participants from the TAU unit were allocated to the TAU group. No group differences were found in key demographical variables (e.g., age, education, and gender ratio), premorbid IQ, psychiatric characteristics (e.g., age of onset, duration of illness, number of hospitalizations, BIS/BAS scores and PANSS 
TABLE 1 | Demographic information.

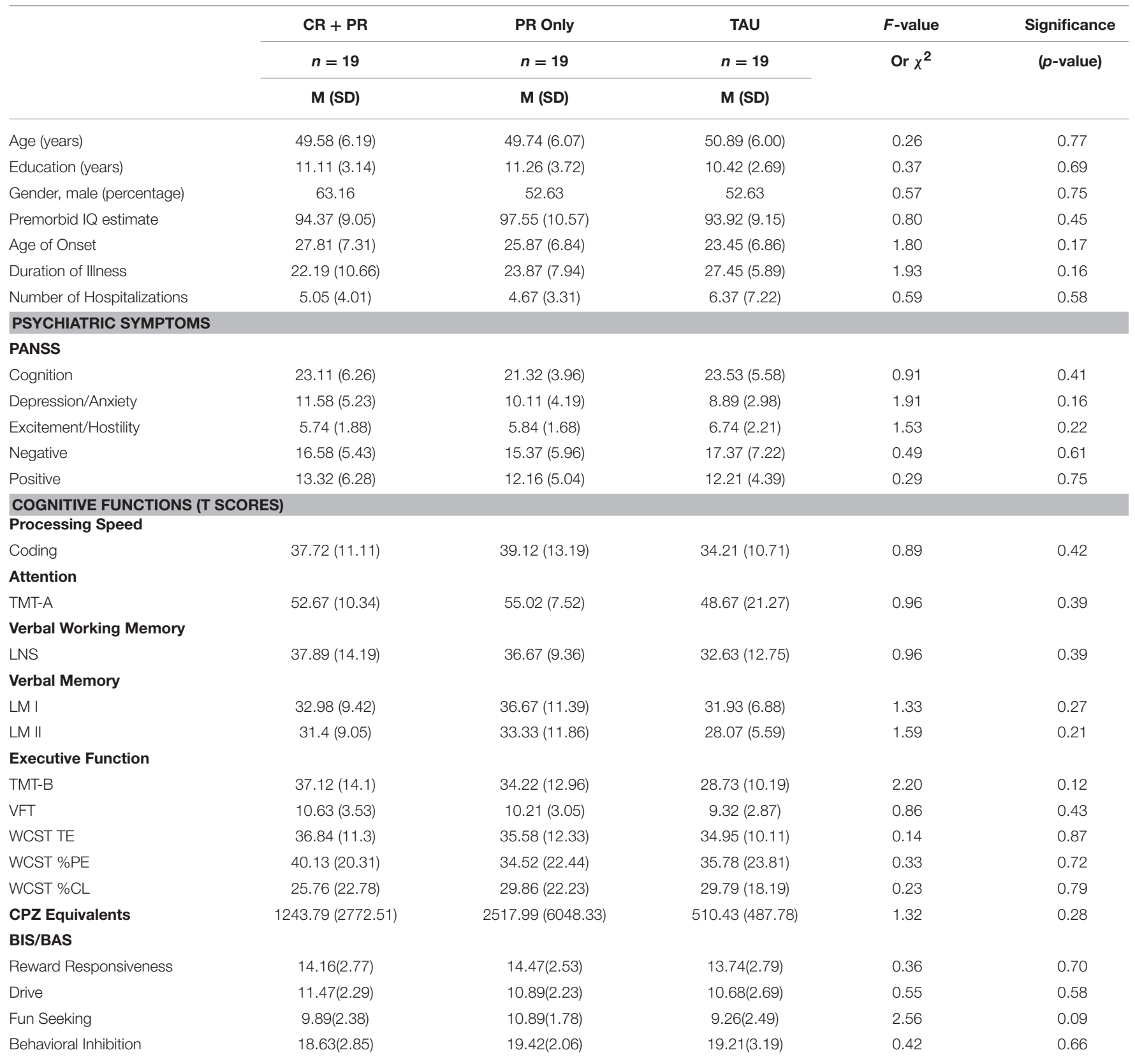

CR, Cognitive remediation; PR, Psychiatric rehabilitation; TAU, Treatment as usual; M, mean; SD, standard deviation; PANSS, Positive and Negative Syndrome Scale; TMT-A, Trail Making Test-A; LNS, Letter-Number Sequencing; LM I, Logical Memory I; LM II, Logical Memory Il; TMT-B, Trail Making Test-B; VFT, Verbal fluency test; WCST TE, Wisconsin Card Sorting Test Total Errors; WCST \%PE, Wisconsin Card Sorting Test Perseveration errors; WCST \%CL, Wisconsin Card Sorting Test Conceptual level responses; CPZ, chlorpromazine.

scores), or baseline neurocognitive functioning (Table 1). The drop-out rates were low for the $\mathrm{CR}+\mathrm{PR}$ (5.3\%) and $\mathrm{PR}$ $(0.0 \%)$ participants, while it was $15.8 \%$ for the TAU participants (Figure 1).

\section{Effects of CR on Neurocognition}

The data for all neurocognitive variables are presented in Table 2. The time (i.e., pre- and post-treatment) $\times$ group (i.e., $\mathrm{CR}$ + PR, PR only, and TAU) interactions were significant for both immediate recall $\left(p<0.001, \eta_{p}^{2}=0.21\right)$ and delayed recall $\left(p=0.03, \eta_{p}^{2}=0.13\right.$ ), with medium to large effect sizes. The total errors, perseverative errors, and conceptual level (CL) responses (\%) were analyzed to observe abstract thinking, learning strategies, and cognitive flexibility. The time $\times$ group interactions were significant for WCST total errors $\left(p=0.02, \eta_{p}^{2}=0.15\right)$ and CL responses $\left(p=0.004, \eta_{p}^{2}\right.$ $=0.20$ ), with medium to large effect sizes (Table 2). No group differences were found for processing speed (WAIS-IV 
TABLE 2 | Effects of cognitive remediation on neurocognition.

\begin{tabular}{|c|c|c|c|c|c|c|c|c|c|}
\hline \multirow[t]{3}{*}{ Outcome measures } & & \multicolumn{2}{|c|}{$\begin{array}{c}\text { CR + PR } \\
n=18\end{array}$} & \multicolumn{2}{|c|}{$\begin{array}{l}\text { PR Only } \\
n=19\end{array}$} & \multicolumn{2}{|c|}{$\begin{array}{c}\text { TAU } \\
n=16\end{array}$} & \multirow{3}{*}{$\frac{\text { Group } \times \text { Time }}{p \text {-value }}$} & \multirow{3}{*}{$\begin{array}{l}\text { ES } \\
\left(\eta_{p}^{2}\right)\end{array}$} \\
\hline & & Pre & Post & Pre & Post & Pre & Post & & \\
\hline & & M (SD) & M (SD) & M (SD) & M (SD) & M (SD) & M (SD) & & \\
\hline \multicolumn{10}{|l|}{ PROCESSING SPEED } \\
\hline & TMT-A & $52.81(10.62)$ & $55.58(12.03)$ & $55.02(7.52)$ & $57.76(6.51)$ & $47.98(22.79)$ & $46.00(22.94)$ & 0.52 & 0.03 \\
\hline \multicolumn{10}{|c|}{ VERBAL WORKING MEMORY } \\
\hline & LNS & 38.89 (13.91) & $41.30(10.91)$ & 36.67 (9.36) & $39.12(10.23)$ & $31.25(13.44)$ & 29.38 (10.63) & 0.16 & 0.07 \\
\hline \multicolumn{10}{|l|}{ VERBAL MEMORY } \\
\hline & LM I & $33.15(9.67)$ & 41.30 (13.19) & $36.67(11.39)$ & $37.02(11.49)$ & 32.08 (7.39) & $29.79(9.23)$ & $<0.01$ & 0.21 \\
\hline & VFT & $10.83(3.52)$ & $10.83(3.4)$ & $10.21(3.05)$ & $10.79(2.49)$ & $9.00(2.97)$ & $9.69(3.16)$ & 0.59 & 0.02 \\
\hline & WCST TE & $36.17(11.23)$ & 30.67 (12.33) & $35.58(12.33)$ & $38.53(8.74)$ & 36.81 (9.57) & 39.63 (9.79) & 0.02 & 0.15 \\
\hline & WCST \%PE & $40.48(20.84)$ & 34.11 (21.99) & $34.52(22.44)$ & $39.14(20.94)$ & $39.07(24.56)$ & $44.14(24.07)$ & 0.17 & 0.07 \\
\hline & WCST \%CL & $27.19(22.54)$ & 36.46 (25.62) & $29.86(22.23)$ & $19.98(16.15)$ & $26.98(17.69)$ & $20.21(17.38)$ & $<0.01$ & 0.20 \\
\hline
\end{tabular}

CR, Cognitive remediation; PR, Psychiatric rehabilitation; TAU, Treatment as usual; ES, Effect size; $\eta_{p}^{2}$, Partial eta square; M, mean; SD, standard deviation; TMT-A, Trail Making Test-A;

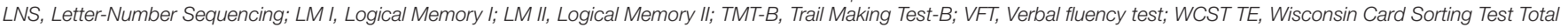
Errors; WCST \%PE, Wisconsin Card Sorting Test Perseveration errors; WCST \%CL, Wisconsin Card Sorting Test Conceptual level responses.

Coding), attention (TMT-A), verbal working memory (WAISIV Letter-Number Sequencing), or cognitive flexibility (WCST perseveration errors).

Least Significant Difference post-hoc tests were conducted on the variables with significant time $\times$ group interactions (i.e., LM I \& II, WCST total errors, and WCST \%CL) to investigate whether the groups differed at post-treatment (Supplemental Tables 1, 2). The CR + PR group showed greater post-treatment performance on both LM I and II compared with the performance of the TAU group (LM I: mean difference $=11.50, p=0.005$; LM II: mean difference $=11.62, p=0.007$ ), while the CR $+\mathrm{PR}$ and PR-only groups did not differ in both LM I and II. The PR-only group showed greater performance at a trend level of significance compared with the TAU group (LM I: mean difference $=7.23$, $p=0.070$; LM II: mean difference $=6.86, p=0.098$ ).

In addition, the $\mathrm{CR}+\mathrm{PR}$ group showed greater posttreatment performance on both WCST total errors and WCST \%CL compared with the PR-only group (WCST total errors: mean difference $=12.28, p=0.026$; WCST \%CL: mean difference $=16.47, p=0.017$ ) and TAU group (WCST total errors: mean difference $=14.00, p=0.015$; WCST $\%$ CL: mean difference $=$ $16.24, p=0.023)$. However, no group differences were found on WCST total errors and WCST \%CL between the PR-only and TAU groups (Figure 3).

\section{Effects of CR in Psychiatric Symptoms}

No significant group differences were observed for psychiatric symptoms (PANSS scores) (Table 3 ).

\section{$\mathbf{R C I}$}

The RCIs were calculated for LM I and II, WCST total errors, and WCST \%CL, which showed significant time $\times$ group interactions. As recommended by Wise (Wise, 2004), RCI scores $\geq 1.96$ were considered reliable changes. As shown in Figure 4, more participants in the $\mathrm{CR}+\mathrm{PR}$ group showed clinically significant improvements for the LM I (CR + PR: 7 participants; PR only: 1 participant; TAU: 2 participants) and LM II (CR + PR: 6 participants; PR only: 2 participants; TAU: 2 participants) (LM I: $\chi^{2}=8.35, p=0.02$; LM II: $\left.\chi^{2}=6.92, p=0.03\right)$. Differences with trend levels of significance were found for WCST total errors (CR + PR: 4 participants; PR only: 0 participants; TAU: 1 participant) and WCST \%CL (CR + PR: 3 participants; PR only: 0 participants; TAU: 0 participants) (WCST total errors: $\chi^{2}=5.18$, $p=0.08$; WCST \%CL: $\left.\chi^{2}=5.67, p=0.06\right)$.

\section{DISCUSSION}

The current study aimed to investigate whether CR treatment within the context of PR treatment $(C R+P R)$ would produce meaningful improvements in neurocognition and psychiatric symptoms in middle-aged or older inpatients with chronic schizophrenia compared to either the PR-only or TAU group. For the $\mathrm{CR}$, we employed a highly personalized and computerized CR protocol with motivational enhancements through a bridging group and motivational interviewing. To the best of our knowledge, the current study is the first RCT of middle-aged or older inpatients with chronic schizophrenia compared with 


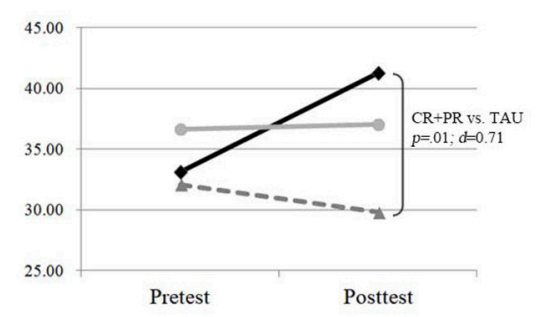

WMS Logical Memory, Immediate

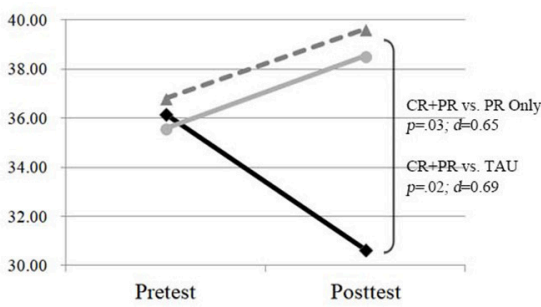

WCST Total Errors

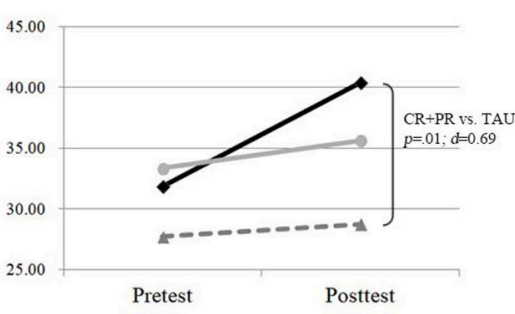

WMS Logical Memory, Delayed

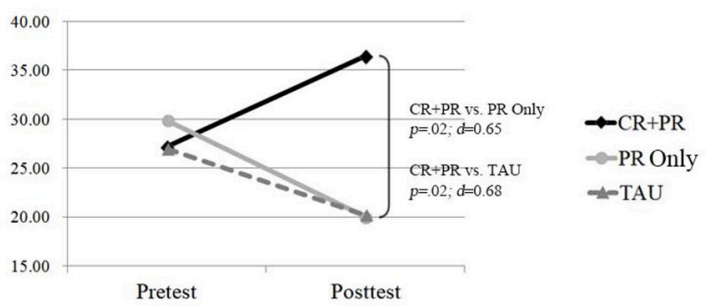

WCST Conceptual Level Responses

FIGURE 3 | Effects of CR on logical memory and executive functioning

TABLE 3 | Effects of cognitive remediation on psychiatric symptoms.

\begin{tabular}{|c|c|c|c|c|c|c|c|c|}
\hline \multirow[t]{3}{*}{ Psychiatric symptoms } & \multicolumn{2}{|c|}{$\begin{array}{c}C R+P R \\
n=18\end{array}$} & \multicolumn{2}{|c|}{$\begin{array}{l}\text { PR Only } \\
n=19\end{array}$} & \multicolumn{2}{|c|}{$\begin{array}{c}\text { TAU } \\
n=16\end{array}$} & \multirow{3}{*}{$\begin{array}{c}\text { Group } \times \text { Time } \\
p \text {-value }\end{array}$} & \multirow{3}{*}{$\begin{array}{l}\mathrm{ES} \\
\left(\eta_{p}^{2}\right)\end{array}$} \\
\hline & Pre & Post & Pre & Post & Pre & Post & & \\
\hline & M (SD) & M (SD) & M (SD) & M (SD) & M (SD) & M (SD) & & \\
\hline \multicolumn{9}{|l|}{ PANSS } \\
\hline Excitement/Hostility & $5.74(1.88)$ & $5.95(2.44)$ & $5.84(1.68)$ & $6.05(1.9)$ & $6.74(2.21)$ & $6.95(4.36)$ & 1.00 & 0.00 \\
\hline Negative & $16.58(5.43)$ & $15.58(7.43)$ & 15.37 (5.96) & $19.05(6.07)$ & $17.37(7.22)$ & $17.68(10.07)$ & 0.12 & 0.08 \\
\hline Positive & $13.32(6.28)$ & $12.26(6.13)$ & $12.16(5.04)$ & $12.53(5.49)$ & $12.21(4.39)$ & 11.05 (6.32) & 0.58 & 0.02 \\
\hline
\end{tabular}

CR, Cognitive remediation; PR, Psychiatric rehabilitation; TAU, Treatment as usual; ES, Effect size; $\eta_{p}^{2}$, Partial eta square; M, mean; SD, standard deviation; PANSS, Positive and Negative Syndrome Scale.

PR-only and TAU groups, especially conducted in a non-western country.

The results of the current study partially supported our primary hypothesis. Specifically, compared with the PR-only and TAU groups, the $\mathrm{CR}+\mathrm{PR}$ group showed greater improvement in executive functioning (e.g., abstract thinking and learning strategies). In addition, the $\mathrm{CR}+\mathrm{PR}$ group showed greater improvements in immediate and delayed LM compared with the TAU group but not the PR-only group. The PR-only group also showed greater improvements in immediate and delayed LM compared with the TAU group. The PR group improved more in executive functioning than the TAU did, but the difference was not reach significant. Importantly, the RCIs indicated that more participants in the $\mathrm{CR}+\mathrm{PR}$ group had clinically meaningful improvements in
LM and executive functioning compared with participants in the PR-only and TAU groups. However, since TAU group was not randomly assigned, the differences between " $\mathrm{CR}+\mathrm{PR}$ vs. TAU" or "PR-only vs. TAU" should not be interpreted as causation.

These findings suggest that CR has additional treatment benefits for executive-level operation when it is delivered within a larger context of PR compared with PR only. Inpatient PR has nonspecific treatment effects on neuropsychological functioning, even without explicit CR training (Spaulding et al., 1999b). In addition to the nonspecific treatment effects of $\mathrm{PR}, \mathrm{CR}$ effectively stimulates neurocognition to synergistically accelerate the benefits of PR.

No group differences were found in other neurocognitive domains or psychiatric symptoms. The pattern of treatment 


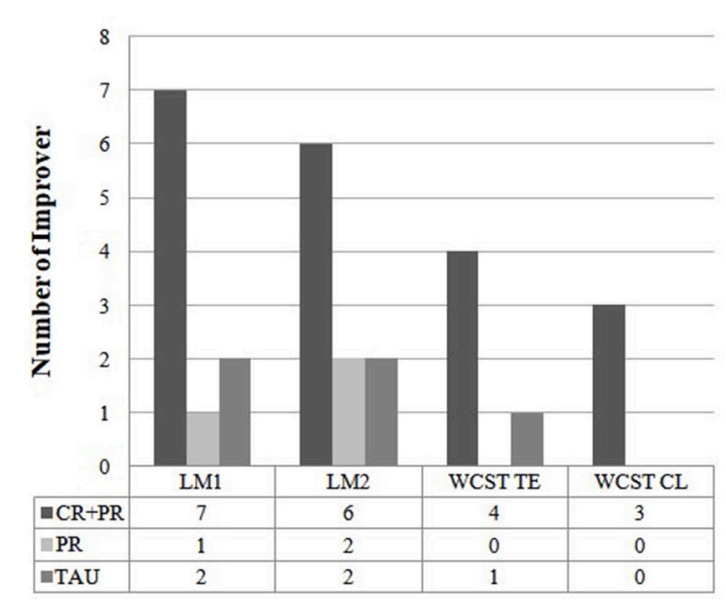

FIGURE 4 | Comparisons of the number of participants with meaningful improvement.

gains found in the current study was somewhat similar to the results of a previous controlled outcome trial that targeted younger patients in a comprehensive and intensive inpatient PR setting (Spaulding et al., 1999a) and that reported significant treatment gains in Card Sort random errors but not perseverative errors, Trails B, and PANSS total and subscales. The null effects of $\mathrm{CR}$ in other neurocognitive domains and psychiatric symptoms could be interpreted in several ways. All participants in the current trial received optimal pharmacotherapy, supportive therapies, and case management, which resulted in nonspecific treatment gains in neurocognition and/or psychiatric symptoms. In addition, the relatively low dose of CR (i.e., 24 sessions, twice per week) and small sample (i.e., 19 per group) in the current study might have resulted in insufficient power for detecting any potential treatment gains.

Comparing the current findings with the results of a previous RCT that was conducted in outpatients with similar ages (Dickinson et al., 2010) shows that, unlike the previous study, the current study found treatment gains in untrained neuropsychological assessments (i.e., executive functioning). Important differences between these two studies need to be acknowledged when interpreting the results. First, the patients examined in the current study were chronic inpatients who participated in the usual inpatient PR-only (without CR) and TAU units. Compared to outpatient clinics, inpatient settings have less cognitive stimulation. Thus, the addition of CR to inpatient $\mathrm{PR}$ might result in additional improvements in neuropsychological assessments compared with control conditions. Second, unlike Dickinson et al. (2010), we did not include active computer skills training as a control condition, and this might have resulted in greater differences between the $\mathrm{CR}+\mathrm{PR}$ and PR-only groups in our study compared with those in the study by Dickinson et al. (2010).

The effects of CR on the neuropsychological assessments in the middle-aged or older inpatients with chronic schizophrenia in the current study were in line with the outcomes of previous $\mathrm{CR}$ studies. Bowie et al. have reported that both younger (early-course group) and older outpatients (longer-course group) with schizophrenia improved in working memory (digit sequencing) and executive functioning (Tower of London), but only the improvements in the early-course group in executive functioning were statistically significant and the improvements in working memory trended to significance. These findings suggested that older inpatients with schizophrenia maintain neuronal plasticity. Thus, CR should be considered as an adjunct treatment to usual PR services.

This study had several limitations. The CR training sessions were designed to have a relatively low dose (i.e., 24 sessions, twice per week for about 3 months) due to the durations of inpatient hospitalizations. Even though the low CR dose was similar to those used in previous studies (van der Gaag et al., 2002; Lindenmayer et al., 2008), more intense CR training might have produced better treatment outcomes. We did not have follow-up assessments also because of the durations of inpatient hospitalizations. Thus, the durability of the treatment gains should be explored in an inpatient PR setting in a longer future study. We should also acknowledge that the half of our evaluators were not blinded completely in measuring psychiatric symptoms or neurocognition in this study. To minimize potential evaluators' bias, all evaluators were trained intensively to approximate a great inter-rater reliability. During the administration and scoring process, they were observed and supervised with verbatim process by licensed psychologists (KHC, WHL, SC). Nevertheless, the evaluators' bias cannot be negated. Importantly, even though our sample size was modest for detecting medium effects, the statistical power of this study might have been insufficient for detecting small to moderate treatment gains. Thus, additional studies with more patients and longer $\mathrm{CR}$ sessions should be conducted to attempt to replicate the current findings. Finally, the effects of such improvements in cognitive function on daily function or social functioning in the actual ward need further investigation.

Despite the limitations noted above, the results of the current study highlight the importance of delivering CR within a PR context to middle-aged or older inpatients with chronic schizophrenia, especially to improve their executive functioning, which is a critical factor for learning in PR and treatment (Green, 1996; Spaulding et al., 1999a; Bowie and Harvey, 2006).

\section{AUTHOR CONTRIBUTIONS}

K-HC, S-HL, and T-YH designed the study. K-HC wrote the first draft of the manuscript and supervised cognitive remediation sessions, S-MK and JK administered cognitive remediation. S-MK, JK, and KP undertook the statistical analysis. S-CP supervised participants' recruitment and administration of psychotropic medications during trials. $\mathrm{W}-\mathrm{HL}$ and SC supervised neurocognitive and clinical 
assessments. All the authors commented on the manuscript. All the authors contributed to and have approved the final manuscript.

\section{FUNDING}

This study was funded by a Yongin Mental Hospital Research and Development Grant to T-YH. The sponsor had no role in the planning or conduct of the study or in the interpretation of the study results.

\section{SUPPLEMENTARY MATERIAL}

The Supplementary Material for this article can be found online at: https://www.frontiersin.org/articles/10.3389/fpsyg. 2017.02364/full\#supplementary-material

\section{REFERENCES}

American Psychiatric Association (1994). Task Force on DSM-IV. Diagnostic and Statistical Manual of Mental Disorders: DSM-IV, 4th Edn. Washington, DC: American Psychiatric Association. xxvii, 886.

Arbuthnott, K., and Frank, J. (2000). Trail making test, part B as a measure of executive control: validation using a set-switching paradigm. J. Clin. Exp. Neuropsychol. 22, 518-528. doi: 10.1076/1380-3395(200008)22:4;1-0;FT518

Bartels, S. J., and Pratt, S. (2009). Psychosocial rehabilitation and quality of life for older adults with serious mental illness: recent findings and future research directions. Curr. Opin. Psychiatry 22, 381-385. doi: 10.1097/YCO.0b013e32832c9234

Bartels, S. J., Pratt, S. I., Mueser, K. T., Forester, B. P., Wolfe, R., Cather, C., et al. (2014). Long-term outcomes of a randomized trial of integrated skills training and preventive healthcare for older adults with serious mental illness. Am. J. Geriatr. Psychiatry 22, 1251-1261. doi: 10.1016/j.jagp.2013.04.013

Bell, M. D., Choi, K.-H., Dyer, C., and Wexler, B. E. (2014). Benefits of cognitive remediation and supported employment for schizophrenia patients with poor community functioning. Psychiatr. Serv. 65, 469-475. doi: 10.1176/appi.ps.201200505

Bell, M. D., Fiszdon, J., Greig, T., Wexler, B., and Bryson, G. (2007). Neurocognitive enhancement therapy with work therapy in schizophrenia: 6-month followup of neuropsychological performance. J. Rehabil. Res. Dev. 44, 761-770. doi: 10.1682/JRRD.2007.02.0032

Bell, M. D., Lysaker, P. H., Beam-Goulet, J. L., Milstein, R. M., and Lindenmayer, J.-P. (1994). Five-component model of schizophrenia: assessing the factorial invariance of the positive and negative syndrome scale. Psychiatry Res. 52, 295-303. doi: 10.1016/0165-1781(94)90075-2

Boron, J. B., Willis, S. L., and Schaie, K. W. (2007). Cognitive training gain as a predictor of mental status. J. Gerontol. B Psychol. Sci. Soc. Sci. 62, 45-52. doi: 10.1093/geronb/62.1.P45

Bowie, C. R., Grossman, M., Gupta, M., Oyewumi, L., and Harvey, P. D. (2014). Cognitive remediation in schizophrenia: efficacy and effectiveness in patients with early versus long-term course of illness. Early Interv. Psychiatry 8, 32-38. doi: 10.1111/eip.12029

Bowie, C. R., and Harvey, P. D. (2006). Cognitive deficits and functional outcome in schizophrenia. Neuropsychiatr. Dis. Treat. 2, 531-536. doi: $10.2147 /$ nedt.2006.2.4.531

Bracy, O. (2012). PSSCogRehab 2012. Indianapolis, IN: Psychological Software Services, Inc.,.

Carver, C. S., and White, T. L. (1994). Behavioral inhibition, behavioral activation, and affective responses to impending reward and punishment: the BIS/BAS Scales. J. Pers. Soc. Psychol. 67, 319-333. doi: 10.1037/0022-3514.67.2.319

Corbera, S., Wexler, B. E., Poltorak, A., Thime, W. R., and Kurtz, M. M. (2017). Cognitive remediation for adults with schizophrenia: does age matter? Psychiatry Res. 247, 21-27. doi: 10.1016/j.psychres.2016.10.084

Dickinson, D., Tenhula, W., Morris, S., Brown, C., Peer, J., Spencer, K., et al. (2010). A randomized, controlled trial of computer-assisted cognitive remediation for schizophrenia. Am. J. Psychiatry 167, 170-180. doi: 10.1176/appi.ajp.2009.09020264

Faul, F., Erdfelder, E., Buchner, A., and Lang, A.-G. (2009). Statistical power analyses using $\mathrm{G}^{*}$ Power 3.1: tests for correlation and regression analyses. Behav. Res. Methods 41, 1149-1160. doi: 10.3758/BRM.41. 4.1149

Fiszdon, J. M., Kurtz, M. M., Choi, J., Bell, M. D., and Martino, S. (2016). Motivational interviewing to increase cognitive rehabilitation adherence in schizophrenia. Schizophr. Bull. 42, 327-334. doi: 10.1093/schbul/sbv143

Granholm, E., McQuaid, J. R., McClure, F. S., Auslander, L. A., Perivoliotis, D., Pedrelli, P., et al. (2005). A randomized, controlled trial of cognitive behavioral social skills training for middle-aged and older outpatients with chronic schizophrenia. Am. J. Psychiatry 162, 520-529. doi: 10.1176/appi.ajp.162.3.520

Green, M. F. (1996). What are the functional consequences of neurocognitive deficits in schizophrenia? Am. J. Psychiatry 153, 321-330.

Green, M. F., Kern, R. S., and Heaton, R. K. (2004). Longitudinal studies of cognition and functional outcome in schizophrenia: implications for MATRICS. Schizophr. Res. 72, 41-51. doi: 10.1016/j.schres.2004.09.009

Green, M. F., and Nuechterlein, K. H. (1999). Should schizophrenia be treated as a neurocognitive disorder? Schizophr. Bull. 25, 309-319.

Grynszpan, O., Perbal, S., Pelissolo, A., Fossati, P., Jouvent, R., Dubal, S., et al. (2011). Efficacy and specificity of computer-assisted cognitive remediation in schizophrenia: a meta-analytical study. Psychol. Med. 41, 163-173. doi: 10.1017/S0033291710000607

Inada, T., and Inagaki, A. (2015). Psychotropic dose equivalence in Japan. Psychiatry Clin. Neurosci. 69, 440-447. doi: 10.1111/pcn.12275

Kay, S. R., Fiszbein, A., and Opler, L. A. (1987). The positive and negative syndrome scale (PANSS) for schizophrenia. Schizophr. Bull. 13, 261-276. doi: $10.1093 /$ schbul/13.2.261

Keefe, R. S. E., and Harvey, P. D. (2012). "Cognitive impairment in schizophrenia," in Handbook of Experimental Pharmacology: Vol. 213, Novel Antischizophrenia Treatments, eds M. A. Geyer and G. Gross (Berlin; Heidelberg: SpringerVerlag), 11-37.

Keefe, R. S., Perkins, D. O., Gu, H., Zipursky, R. B., Christensen, B. K., and Lieberman, J. A. (2006). A longitudinal study of neurocognitive function in individuals at-risk for psychosis. Schizophr. Res. 88, 26-35. doi: 10.1016/j.schres.2006.06.041

Kim, K.-H., and Kim, W.-S. (2001). Korean-BAS/BIS Scale. Korean J. Health Psychol. 6, 19-37.

Kim, S.-G., Lee, E.-H., Hwang, S.-T., Park, K., Chey, J., Hong, S.-H., et al. (2015). Estimation of K-WAIS-IV Premorbid Intelligence in South Korea: development of the KPIE-IV. Clin. Neuropsychol. 29, 19-29. doi: 10.1080/13854046.2015.1072248

Kontis, D., Huddy, V., Reeder, C., Landau, S., and Wykes, T. (2013). Effects of age and cognitive reserve on cognitive remediation therapy outcome in patients with schizophrenia. Am. J. Geriatr. Psychiatry 21, 218-230. doi: 10.1016/j.jagp.2012.12.013

Krabbendam, L., and Aleman, A. (2003). Cognitive rehabilitation in schizophrenia: a quantitative analysis of controlled studies. Psychopharmacology (Berl) 169, 376-382. doi: 10.1007/s00213-002-1326-5

Kreiner, D. S., and Ryan, J. J. (2001). Memory and motor skill components of the WAIS-III Digit Symbol-Coding subtest. Clin. Neuropsychol. 15, 109-113. doi: 10.1076/clin.15.1.109.1906

Kurtz, M. M., Moberg, P. J., Ragland, J. D., Gur, R. C., and Gur, R. E. (2005). Symptoms versus neurocognitive test performance as predictors of psychosocial status in schizophrenia: a 1-and 4-year prospective study. Schizophr. Bull. 31, 167-174. doi: 10.1093/schbul/sbi004

Lee, H., Jang, S., Lee, G., Park, S., Medalia, A., and Choi, K. (2017). Informationally administered reward enhances intrinsic motivation in schizophrenia. Psychiatry Res. 256, 290-297. doi: 10.1016/j.psychres.2017.06.049 
Lee, J. H., Lee, K. U., Lee, D. Y., Jhoo, J. H., Kim, K. W., and Woo, J. I. (2000). Korean version of the consortium to establish a registry for Alzheimer's disease assessment packet (CERAD-K): clinical and neuropsychological assessment batteries. Neurobiol. Aging 21:32. doi: 10.1016/S0197-4580(00)82818-3

Lindenmayer, J.-P., McGurk, S. R., Mueser, K. T., Khan, A., Wance, D., Hoffman, L., et al. (2008). A randomized controlled trial of cognitive remediation among inpatients with persistent mental illness. Psychiatr. Serv. 59, 241-247. doi: 10.1176/ps.2008.59.3.241

Lysaker, P. H., and Buck, K. D. (2007). Neurocognitive deficits as a barrier to psychosocial function in schizophrenia: effects on learning, coping, and self-concept. J. Psychosoc. Nurs. Ment. Health Serv. 45, 24-30.

McGurk, S. R., and Mueser, K. T. (2008). Response to cognitive rehabilitation in older versus younger persons with severe mental illness. Am. J. Psychiatr. Rehabil. 11, 90-105. doi: 10.1080/15487760701853136

McGurk, S. R., Mueser, K. T., Feldman, K., Wolfe, R., and Pascaris, A. (2007a). Cognitive training for supported employment: 2-3 year outcomes of a randomized controlled trial. Am. J. Psychiatry 164, 437-441. doi: 10.1176/ajp.2007.164.3.437

McGurk, S. R., Mueser, K. T., Xie, H., Welsh, J., Kaiser, S., Drake, R. E., et al. (2015). Cognitive enhancement treatment for people with mental illness who do not respond to supported employment: a randomized controlled trial. Am. J. Psychiatry 172, 852-861. doi: 10.1176/appi.ajp.2015.14030374

McGurk, S. R., Twamley, E. W., Sitzer, D. I., McHugo, G. J., and Mueser, K. T. (2007b). A meta-analysis of cognitive remediation in schizophrenia. Am. J. Psychiatry 164, 1791-1802. doi: 10.1176/appi.ajp.2007.07060906

Medalia, A., Aluma, M., Tryon, W., and Merriam, A. E. (1998). Effectiveness of attention training in schizophrenia. Schizophr. Bull. 24, 147-152. doi: 10.1093/oxfordjournals.schbul.a033306

Medalia, A., Revheim, N., and Casey, M. (2000). Remediation of memory disorders in schizophrenia. Psychol. Med. 30, 1451-1459. doi: 10.1017/S0033291799002913

Medalia, A., Revheim, N., and Casey, M. (2001). The remediation of problem-solving skills in schizophrenia. Schizophr. Bull. 27, 259-267. doi: 10.1093/oxfordjournals.schbul.a006872

Medalia, A., Revheim, N., and Herlands, T. (2009). Cognitive Remediation for Psychological Disorders: Therapist Guide. Newyork: Oxford University Press.

Ministry of Health and Welfare (2016). Mental Health Statistics: Pilot Study. Seoul: National Mental Health Center.

Owen, A. M., Hampshire, A., Grahn, J. A., Stenton, R., Dajani, S., Burns, A. S., et al. (2010). Putting brain training to the test. Nature 465, 775-778. doi: $10.1038 /$ nature09042

Rund, B. R., Melle, I., Friis, S., Johannessen, J. O., Larsen, T. K., Midbøe, L. J., et al. (2007). The course of neurocognitive functioning in first-episode psychosis and its relation to premorbid adjustment, duration of untreated psychosis, and relapse. Schizophr. Res. 91, 132-140. doi: 10.1016/j.schres.2006.11.030

Sartory, G., Zorn, C., Groetzinger, G., and Windgassen, K. (2005). Computerized cognitive remediation improves verbal learning and processing speed in schizophrenia. Schizophr. Res. 75, 219-223. doi: 10.1016/j.schres.2004.10.004

Schoepf, D., Uppal, H., Potluri, R., and Heun, R. (2014). Physical comorbidity and its relevance on mortality in schizophrenia: a naturalistic 12-year follow-up in general hospital admissions. Eur. Arch. Psychiatry Clin. Neurosci. 264, 3-28. doi: 10.1007/s00406-013-0436-x

Sharma, I., Srivastava, J., Kumar, A., and Sharma, R. (2016). Cognitive remediation therapy for older adults. J. Geriatr. Mental Health 3, 57-65. doi: 10.4103/2348-9995.181919

Silverstein, S. M., Hatashita-Wong, M., Solak, B. A., Uhlhaas, P., Landa, Y., Wilkniss, S. M., et al. (2005). Effectiveness of a two-phase cognitive rehabilitation intervention for severely impaired schizophrenia patients. Psychol. Med. 35, 829-837. doi: 10.1017/S0033291704003356

Silverstein, S. M., Spaulding, W. D., Menditto, A. A., Savitz, A., Liberman, R. P., Berten, S., et al. (2009). Attention shaping: a reward-based learning method to enhance skills training outcomes in schizophrenia. Schizophr. Bull. 35, 222-232. doi: $10.1093 / \mathrm{schbul} / \mathrm{sbm} 150$

Smith, T. E., Hull, J. W., Goodman, M., Hedayat-Harris, A., Willson, D. F., Israel, L. M., et al. (1999). The relative influences of symptoms, insight, and neurocognition on social adjustment in schizophrenia and schizoaffective disorder. J. Nerv. Ment. Dis. 187, 102-108. doi: 10.1097/00005053-199902000-00006

Spaulding, W. D., Reed, D., Sullivan, M., Richardson, C., and Weiler, M. (1999a). Effects of cognitive treatment in psychiatric rehabilitation. Schizophr. Bull. 25, 657-676. doi: 10.1093/oxfordjournals.schbul.a033409

Spaulding, W. D., Fleming, S. K., Reed, D., Sullivan, M., Storzbach, D., and Lam, M. (1999b). Cognitive functioning in schizophrenia: implications for psychiatric rehabilitation. Schizophr. Bull. 25, 275-289. doi: 10.1093/oxfordjournals.schbul.a033378

Tsoutsoulas, C., Mulsant, B. H., Kalache, S. M., Kumar, S., Ghazala, Z., Voineskos, A. N., et al. (2016). The influence of medical burden severity and cognition on functional competence in older community-dwelling individuals with schizophrenia. Schizophr. Res. 170, 330-335. doi: 10.1016/j.schres.2015.12.009

Ueland, T., and Rund, B. (2005). Cognitive remediation for adolescents with early onset psychosis: a 1-year follow-up study. Acta Psychiatr. Scand. 111, 193-201. doi: 10.1111/j.1600-0447.2004.00503.x

van der Gaag, M., Kern, R. S., van den Bosch, R. J., and Liberman, R. P. (2002). A controlled trial of cognitive remediation in schizophrenia. Schizophr. Bull. 28, 167-176. doi: 10.1093/oxfordjournals.schbul.a006919

Vauth, R., Corrigan, P. W., Clauss, M., Dietl, M., Dreher-Rudolph, M., Stieglitz, R. D., et al. (2005). Cognitive strategies versus self-management skills as adjunct to vocational rehabilitation. Schizophr. Bull. 31, 55-66. doi: 10.1093/schbul/sbi013

Wechsler, D. (2008). WAIS-IV Technical and Interpretation Manual. San Antonio, TX: Pearson.

Wechsler, D. (2009). Wechsler Memory Scale, Fourth Edition (WMS-IV). New York, NY: Pearson: Psychological Corporation.

Willis, S. L., Tennstedt, S. L., Marsiske, M., Ball, K., Elias, J., Koepke, K. M., et al. (2006). Long-term effects of cognitive training on everyday functional outcomes in older adults. JAMA 296, 2805-2814. doi: 10.1001/jama.296.23.2805

Wise, E. A. (2004). Methods for analyzing psychotherapy outcomes: a review of clinical significance, reliable change, and recommendations for future directions. J. Pers. Assess. 82, 50-59. doi: 10.1207/s15327752jpa8201_10

Wykes, T., Huddy, V., Cellard, C., McGurk, S. R., and Czobor, P. (2011). A meta-analysis of cognitive remediation for schizophrenia: methodology and effect sizes. Am. J. Psychiatry 168, 472-485. doi: 10.1176/appi.ajp.2010.10 060855

Wykes, T., Newton, E., Landau, S., Rice, C., Thompson, N., and Frangou, S. (2007). Cognitive remediation therapy (CRT) for young early onset patients with schizophrenia: an exploratory randomized controlled trial. Schizophr. Res. 94, 221-230. doi: 10.1016/j.schres.2007.03.030

Wykes, T., and Spaulding, W. D. (2011). Thinking about the future cognitive remediation therapy-what works and could we do better? Schizophr. Bull. 37, S80-S90. doi: 10.1093/schbul/sbr064

Conflict of Interest Statement: The authors declare that the research was conducted in the absence of any commercial or financial relationships that could be construed as a potential conflict of interest.

Copyright (C) 2018 Choi, Kang, Kim, Lee, Park, Lee, Choi, Park and Hwang. This is an open-access article distributed under the terms of the Creative Commons Attribution License (CC BY). The use, distribution or reproduction in other forums is permitted, provided the original author(s) and the copyright owner are credited and that the original publication in this journal is cited, in accordance with accepted academic practice. No use, distribution or reproduction is permitted which does not comply with these terms. 